\title{
Tratamiento endourológico de la estenosis pieloureteral en la edad pediátrica: nuestra experiencia
}

\author{
Romero Otero J*, Gómez Fraile A, Blanco Carballo O*, Aransay Bramtot A, López Vázquez F, \\ Lovaco Castellano $\mathrm{F}^{* *}$.
}
Sección de Urología Pediátrica. *Servicio de Urología. Hospital Universitario 12 de Octubre de Madrid. **Servicio de Urología. Hospital Universitario Ramón y Cajal. Madrid.

Actas Urol Esp. 2007;31(2):146-152

\section{RESUMEN}

TRATAMIENTO ENDOUROLÓGICO DE LA ESTENOSIS PIELOURETERAL EN LA EDAD PEDIÁTRICA: NUESTRA EXPERIENCIA Introducción. La estenosis de la unión pieloureteral (EPU) es la causa más frecuente de hidronefrosis en la infancia. El "patrón oro" para su tratamiento es la pieloplastia a cielo abierto, pero cada vez están más en auge los procedimientos mínimamente invasivos. Presentamos nuestra serie de EPU en edad pediátrica tratados endourológicamente y revisamos la literatura.

Material y métodos. Estudio retrospectivo y descriptivo de los niños diagnosticados de EPU, que han recibido tratamiento vía endourológica en nuestro centro, en el intervalo de tiempo comprendido de enero1998-enero2005. Recogemos las variables en 3 periodos según un criterio cronológico: $1^{\circ}$ Prequirúrgico: edad, sexo, tratamiento previo o no, ecografia (ECO) y renograma isotópico diurético con mercaptoacetiltriglicina (MAG-3) previos a la intervención. $2^{\text {o }}$ Quirúrgico: tipo de procedimiento. $3^{\circ}$ Postquirúrgico. tiempo de evolución, recidiva o no y tratamiento de la misma y estado actual valorando estado clínico, ECO y MAG3.

Resultados: 7 niños han sido intervenidos mediante técnica endourológica en este periodo, con un rango de edad de 13 meses- 168 meses. En 5 de ellos se realizó como tratamiento secundario tras pieloplastia abierta, y en dos como tratamiento primario. La ECO previa mostraba: hidronefrosis grado III en 3, y IV en 4(según clasificación de la Sociedad de Urología Fetal) y en el renograma isotópico las curvas de eliminación según el patrón de O’Reilly eran 6 tipo II y 1 tipo IIIb. Se realizaron: 3 endopielotomías percutáneas. Vía retrógrada 3 dilataciones con balón y un Acucise. Con un tiempo medio de seguimiento de 37 meses, se han evidenciado que 5 están asintomáticos y sin evidencia de recidiva ( $71 \%$ de éxito).

Existen 2 recidivas (ambos tratamientos secundarios), uno vía retrógrada (dilatación con balón) y el otro anterógrada.

Conclusión. El tratamiento de la EPU en la edad pediátrica vía endourológica, es factible pero se debe individualizar en cada caso. Estudios prospectivos de mayor tamaño deben ser realizados para poder extraer conclusiones.

Palabras clave: Estenosis pieloureteral. Endourología. Pieloplastia. Acucise. Endopielotomía. Hidronefrosis.

\section{ABSTRACT \\ ENDOUROLOGICAL TREATMENT OF PELVIURETERIC JUNCTION OBSTRUCTION IN PAEDIATRIC PATIENTS: OUR EXPERIENCE}

Endourological treatment of pelviureteric junction obstruction in paediatric patients: our experience.

Introduction. Pelviureteric junction (PUJ) obstruction is the most common cause of hydronephrosis in the infancy. The gold standard of treatment is open pyeloplasty, but there is an ever greater tendency towards minimally invasive procedures. We present our serie of paediatric patients with endourologically treated PUJ obstruction, together with a review of the literature.

Material and methods. Retrospective, descriptive study of the children diagnosed of PUJ obstruction who were treated by an endourological technique in our centre between January 1988 and January 2005. We gathered data on 3 periods of time:1 $1^{\text {st }}$. Presurgical: age, sex, previous treatment, ultrasound (USS) and nuclear medicine (MAG-3) studies; $2^{\text {nd }}$. Surgical: type of procedure; $3^{\text {rd }}$. Surgical: recurrence or not and its treatment, and the current state of the patient.

Results. Seven children, with an age range of 13 months to 14 years, underwent operation using an endourological technique. The treatment was secondary in five of these patients, after open pyeloplasty, and was primary in 2 cases. The preoperative USS showed grade III dilatation in 3 and grade IV dilatation in 4, and the MAG-3 study showed type II curves in 6 and a type IIIb curve in 1 . Three percutaneous endopyelotomies were performed and, by the retrograde approach, 3 balloon dilatations and one Acucise. With a mean follow-up of 37 months, 2 cases of recurrence (both in patients receiving secondary treatment) have been observed, one in whom a retrograde technique (balloon dilatation) was used and the other in a patient treated by an antegrade technique. The remaining five patients are asymptomatic and show no evidence of recurrence ( $71 \%$ of the patients).

Conclusion. The endourological treatment of PUJ obstruction in paediatric patients is possible but must be individualised in each case. Larger, prospective studies need to be performed in order to reach conclusions.

Keywords: Pelviureteric junction obstruction. Endourology. Pyeloplasty. Acucise. Endopyelotomy. Hydronephrosis. 
$\mathrm{L}$ a estenosis de la unión pieloureteral (EPU) puede ser diagnosticada tanto en la infancia como en el adulto. En los niños representa la causa más frecuente de hidronefrosis ${ }^{1}$. En ambas edades la pieloplastia tipo Anderson Hynes a cielo abierto está aceptada como el "patrón oro" para su tratamiento ${ }^{2}$. Pero mientras que en el adulto multitud de procedimientos terapéuticos presentan buenos resultados, en la edad pediátrica la práctica de técnicas endourológicas ha sido un tema controvertido, pues al ser un grupo tan heterogéneo tanto en talla como en edad, existe una desproporción con el material quirúrgico que se ha de emplear. Aportamos nuestra experiencia en endopielotomías para el tratamiento de la EPU en la edad pediátrica.

\section{MATERIAL Y MÉTODOS}

Realizamos un estudio retrospectivo y descriptivo de los individuos en edad pediátrica, diagnosticados de EPU, que han sido tratados mediante una técnica endourológica en nuestro centro, en el intervalo de tiempo comprendido desde enero de 1998 hasta enero del 2005. De su historial clínico hemos recogido los datos dividiéndolos en tres periodos siguiendo un criterio cronológico:

1. Periodo prequirúrgico: Edad, sexo, realización de tratamiento previo (nuestro procedimiento endourológico sería considerado como tratamiento secundario) o no (tratamiento primario), ecografía del aparato urinario (ECO) previa a la intervención (clasificamos los grados de hidronefrosis según la Sociedad de Urología Fetal), renograma isotópico diurético mediante la utilización de mercaptoacetilglicina (MAG3) marcado con tecnecio 99 previo a la cirugía (analizamos las curvas de eliminación del radiofármaco según los patrones de $\mathrm{O}^{`} \mathrm{Reilly}^{3}$ y la función renal diferencial según los grupos de Ransley ${ }^{4}$ ).

2. Periodo quirúrgico: Tipo de intervención endourológica practicada, consideramos la vía de acceso (anterógrada o retrógrada) y el instrumental quirúrgico empleado para solucionar la estenosis propiamente dicha (asa de Collins, balón de dilatación y Acucise).

3. Periodo posquirúrgico: Existencia o no de recidiva y tratamiento de la misma, tiempo de evolución y estado del paciente en la última revi- sión en consulta, donde valoramos: el estado clínico, ECO y MAG3 (en ambas pruebas utilizamos los mismos criterios que en el periodo prequirúrgico).

Las técnicas endourológicas que hemos empleado se pueden dividir en dos grupos en función de la vía de acceso: anterógrada o retrógrada.

Para la vía anterógrada posicionamos al niño en decúbito supino, realizamos una cistoscopia (cistoscopio de 10,5 Fr., que dispone de un canal recto de trabajo), introducimos un catéter ureteral (variable en función de la talla del niño) y procedemos a opacificar la vía urinaria (así comprobamos el correcto emplazamiento del mismo y disponemos de un "dibujo" de la vía urinaria), posteriormente fijamos el catéter a una sonda vesical tipo foley (el tamaño también varía en función de la talla del niño) y para finalizar posicionamos al enfermo en decúbito prono. A continuación con la vía rellena de contraste y opcionalmente de azul de metileno, bajo control fluoroscópico, puncionamos el cáliz inferior y ayudándonos de dilatadores telescópicos (tipo Alken) (Fig. 1) ampliamos el trayecto. Colocamos una guía cebra de seguridad (así siempre tenemos tutorizado el trayecto) y emplazamos un amplatz (es un amplatz casero) que facilita considerablemente el paso de todo tipo de instrumental (resector de 9,5 Fr.). Gracias a la visualización del catéter ureteral localizamos la unión pielo-ureteral y mediante un asa de Collins seccionamos la zona estenótica (Fig. 2) hasta ver grasa perirrenal. Reemplazamos el catéter ureteral por un catéter doble $\mathrm{J}$ y además ayudamos al drenaje de

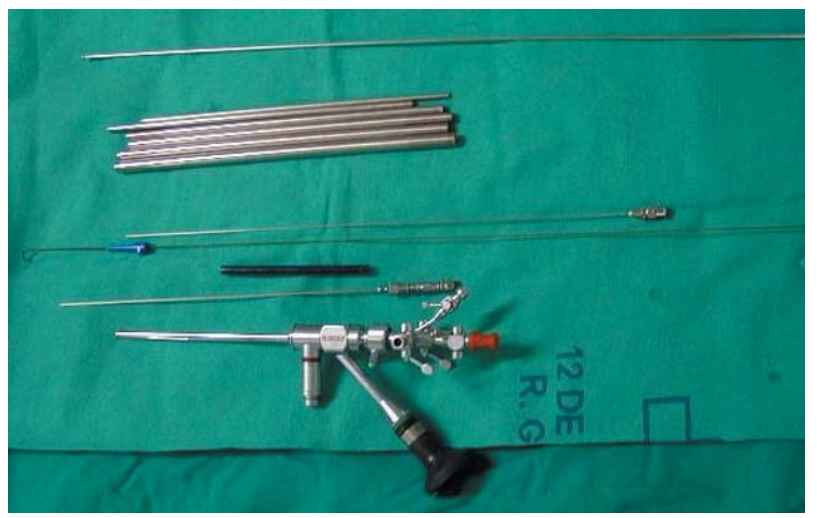

FIGURA 1. Se muestra el resector, los dilatadores de Alken y las guias. 


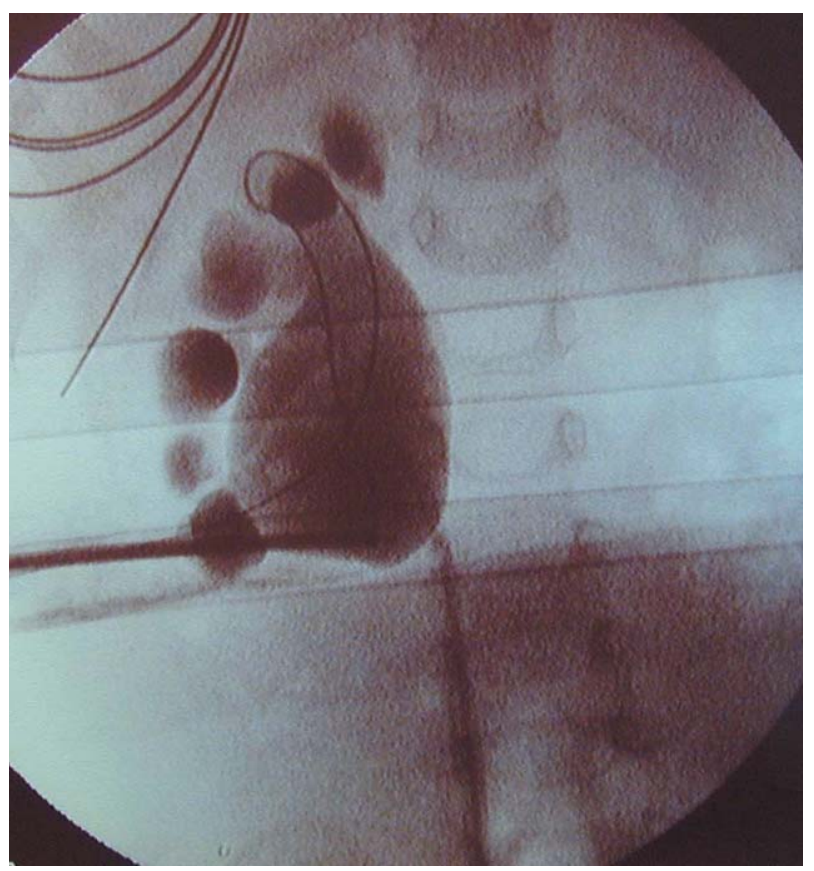

FIGURA 2. Pelvis renal opacificada con relector en su interior. Asi mismo se objetiva le existencia de un catéter ureteral y de una guia de seguridad.

la pelvis renal mediante el emplazamiento de una nefrostomía por el trayecto percutáneo. El doble $\mathrm{J}$ lo retiramos a las 6 semanas de la intervención.

Para la vía retrógrada, colocamos al paciente en decúbito supino, realizamos una cistoscopia (cistoscopio de 10,5 Fr) y así cateterizamos el meato ureteral del riñón afecto con una guía hidrofilica de 0,035 , sobre ella pasamos un catéter ureteral del número 4. Retiramos la guía y esto nos permite introducir contraste para opacificar la vía urinaria. Reintroducimos la guía y retiramos el catéter ureteral, de este modo podemos introducir un Balón de dilatación (Fig. 3), o el Acucise (según procedimiento) (Fig. 4) tutorizado por dicha guía. En el primer caso utilizamos un balón de $2 \mathrm{~cm}$ de longitud por $3 \mathrm{~mm}$ de diámetro, bajo control fluoroscópico colocamos el balón sobre la estenosis y dilatamos el balón a 9 atmósferas de presión, durante 45 segundos un total de tres veces. Retiramos el balón y recolocamos un catéter tipo doble $\mathrm{J}$ del número 4 durante 6 semanas. El otro procedimiento consiste en la introducción de un Acucise del número 5 bajo control fluoroscópico, una vez constatada la correcta colocación del instrumento sobre la estenosis ureteral, realizamos corte a 75 Watios de

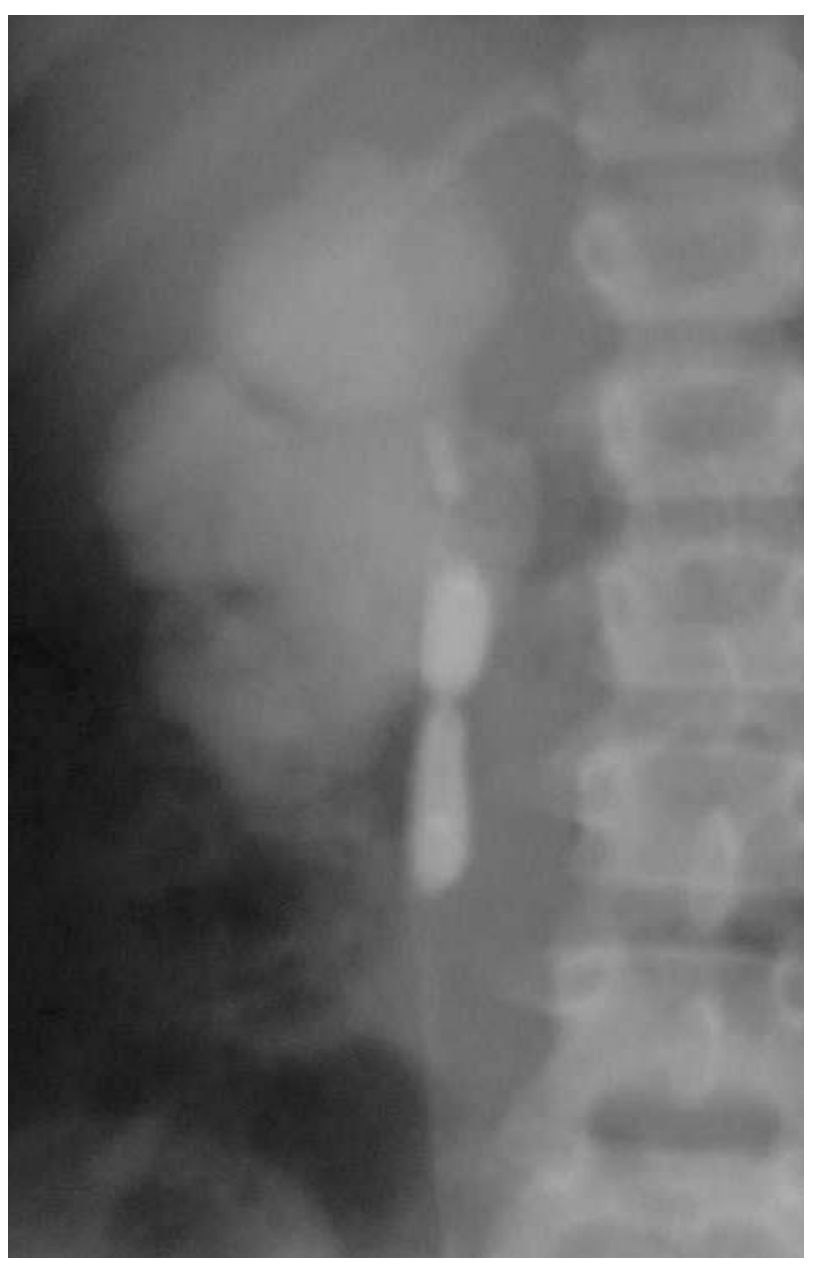

FIGURA 3. Balón de dilatación que presenta una muesca debido a la estenosis de la unión.

potencia hasta que se objetiva la extravasación del contraste. Retiramos el Acucise y dejamos emplazado un catéter tipo doble $\mathrm{J}$ número 4 durante 6 semanas.

\section{RESULTADOS}

En el periodo comprendido entre enero de 1998 y enero del 2005, 7 niños diagnosticados de EPU han sido intervenidos mediante alguna técnica endourológica en nuestro centro; 4 varones y 3 mujeres (Tabla 1). La edad media fue de 61 meses (13-168 meses). En 5 casos se había realizado previamente otro procedimiento terapéutico (pieloplastia tipo Anderson Hynes), y 2 no habían recibido tratamiento previo. En todos ellos se realizó previamente una $\mathrm{ECO}$, que mostraba en 3 niños una dilatación grado III y en 4 grado IV (clasificados según la Sociedad de Urología Fetal), 


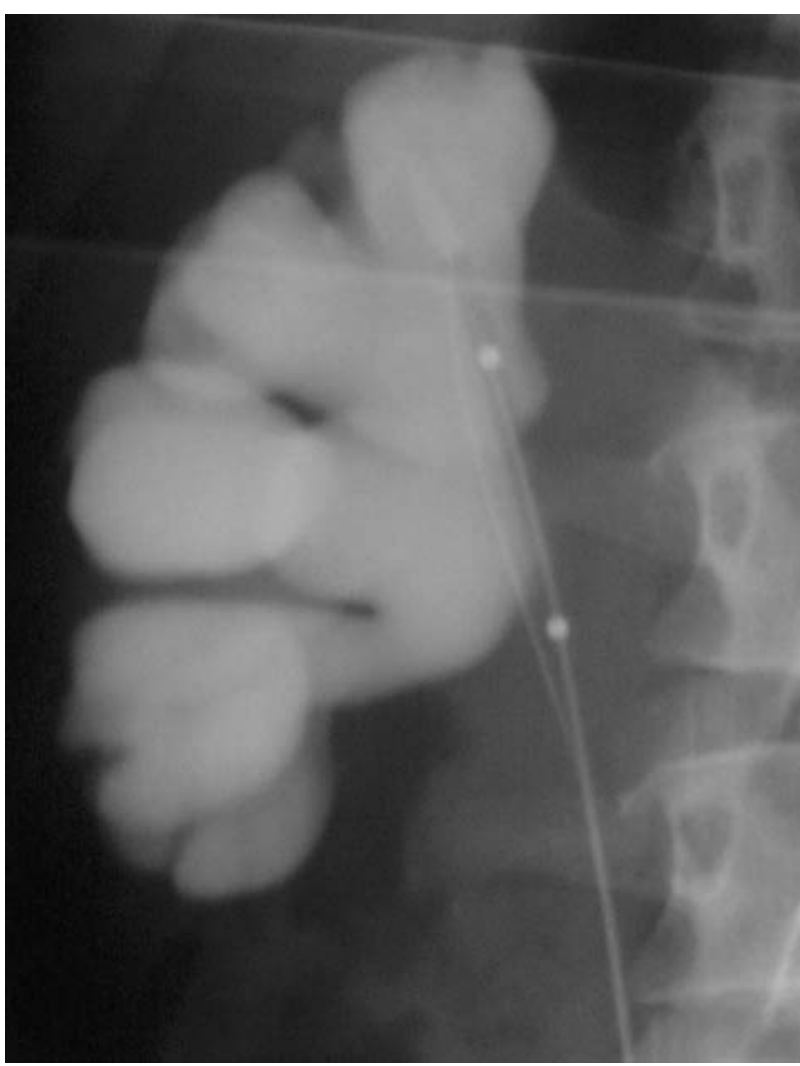

FIGURA 4. Acucise localizado en el segmento de estenosis, se objetiva su correcta posición gracias a las dos marcas radiopacas existentes. así como un MAG-3 en el cual se evidenció: en 6 una curva de eliminación del radiofármaco tipo II (obstructiva) y en 1 tipo IIIb (según patrones de $\mathrm{O}^{\prime}$ Reilly ${ }^{3}$ ).

Como procedimiento terapéutico se practicaron: 3 endopielotomías percutáneas, 3 dilataciones con balón vía retrógrada y en el último un Acucise.

Con un tiempo medio de seguimiento de 37 meses (6-84 meses), 5 de los 7 niños (71\%) están asintomáticos, con un estudio ecográfico y renograma diurético dentro de la normalidad. En los 2 niños restantes ha fallado el procedimiento endourológico, siendo ambos casos de tratamiento secundario (tras realización de pieloplastia). En el primero tras el fracaso de la pieloplastia se realizó una dilatación con balón. $\mathrm{Al}$ año de evolución comenzó con dolor lumbar y en el renograma isotópico diurético se objetivaba una eliminación del MAG-3 siguiendo una curva tipo III-b, por lo que se decidió un nuevo tratamiento endoscópico tipo Acucise. Un año después se encontraba asintomático y en el MAG-3 se constataba una curva de eliminación normal (así en realidad hemos realizado 8 procedimientos endourológicos, aunque dos procedimientos son en un mismo niño). Luego en realidad la tasa de éxito ha sido de 6/8 lo que representa un $75 \%$.

Tabla 1

Resultados de los pacientes intervenidos en nuestro centro

\begin{tabular}{|c|c|c|c|c|c|c|c|}
\hline $\begin{array}{l}\text { Procedimiento } \\
\text { previo }\end{array}$ & ECO & MAG-3 & $\begin{array}{l}\text { Tratamiento } \\
\text { endoscópico }\end{array}$ & $\begin{array}{l}\text { Edad al } \\
\text { tratamiento }\end{array}$ & $\begin{array}{l}\text { Recidiva y } \\
\text { tratamiento }\end{array}$ & $\begin{array}{l}\text { Tiempo } \\
\text { evolución }\end{array}$ & $\begin{array}{l}\text { Estado } \\
\text { Actual }\end{array}$ \\
\hline $\begin{array}{l}\text { Anderson } \\
\text { Hynes }\end{array}$ & III & IIIb & Percutánea & 20 meses & No & 24 meses & $\begin{array}{l}\text { ECO:II } \\
\text { MAG-3:II }\end{array}$ \\
\hline $\begin{array}{l}\text { Anderson } \\
\text { Hynes }\end{array}$ & IV & II & $\begin{array}{l}\text { Dilatación } \\
\text { con balón }\end{array}$ & 84 meses & $\begin{array}{l}\text { Sí } \\
\text { Acucise }\end{array}$ & 12 meses & $\begin{array}{l}\text { ECO:III } \\
\text { MAG-3:IIIa }\end{array}$ \\
\hline $\begin{array}{l}\text { Anderson } \\
\text { Hynes }\end{array}$ & IV & II & $\begin{array}{l}\text { Dilatación } \\
\text { con balón }\end{array}$ & 48 meses & No & 84 meses & $\begin{array}{l}\text { ECO:III } \\
\text { MAG-3:IIIa }\end{array}$ \\
\hline $\begin{array}{l}\text { Anderson } \\
\text { Hynes }\end{array}$ & III & II & $\begin{array}{l}\text { Dilatación } \\
\text { con balón }\end{array}$ & 42 meses & No & 48 meses & $\begin{array}{l}\text { ECO:II } \\
\text { MAG-3:IIIa }\end{array}$ \\
\hline $\begin{array}{l}\text { Anderson } \\
\text { Hynes }\end{array}$ & IV & II & Percutánea & 13 meses & $\begin{array}{l}\text { Sí } \\
\text { Nefrectomía }\end{array}$ & & \\
\hline NO & III & II & Acucise & 168 meses & No & 60 meses & $\begin{array}{l}\text { ECO:III } \\
\text { MAG-3:IIIa }\end{array}$ \\
\hline NO & IV & II & Percutánea & 54 meses & No & 14 meses & $\begin{array}{l}\text { ECO:IV } \\
\text { MAG:IIIa }\end{array}$ \\
\hline $\begin{array}{l}2 \text { primarios } \\
5 \text { secundarios }\end{array}$ & $\begin{array}{l}\text { III:3 } \\
\text { IV:4 }\end{array}$ & $\begin{array}{l}\text { II:6 } \\
\text { IIIb: } 1\end{array}$ & $\begin{array}{l}\text { Percutánea:3 } \\
\text { Dilataciones:3 } \\
\text { Acucise: } 1\end{array}$ & $\begin{array}{l}61 \text { meses } \\
(13-168 \mathrm{~m})\end{array}$ & $\begin{array}{l}\text { No:5 } \\
\text { Si:2 }\end{array}$ & $(12-84 \mathrm{~m})$ & $\begin{array}{l}\text { ECO }: I I=2, I I I=3, I V=1 \\
\text { MAG-3:I=1,IIIa=5 }\end{array}$ \\
\hline
\end{tabular}


El segundo caso fue más complejo, pues se trataba de un niño de 7 meses de edad que se había intervenido mediante una pieloplastia tipo Anderson Hynes. A los 6 meses de evolución presentó un episodio de dolor lumbar y fiebre, por lo que se le realizó, entre otras pruebas, una ECO en la que se visualizaba un riñón pionefrótico, motivo por el cual se procedió a tratamiento médico y drenaje mediante la colocación de una nefrostomía percutánea. Dada la situación del enfermo y aprovechando el trayecto percutáneo ya realizado, cuando el niño se hubo recuperado y completado el tratamiento antibiótico, se decidió la realización de una endopielotomía bajo control nefroscópico. En el primer control postoperatorio presentaba en la ECO una dilatación grado IV, con importante adelgazamiento de la corteza renal, el MAG-3 constataba la existencia de anulación funcional de esa unidad, por lo que se decidió proceder a la nefrectomía.

\section{DISCUSIÓN}

Desde que en 1886 Trendelemburg realizase la primera intervención sobre una EPU con intención desostructiva ${ }^{5}$, muchos son los procedimientos que se han desarrollado, así hoy en día se aceptan: pieloplastia a cielo abierto, endopielotomía vía anterógrada o retrógrada (Acucise), endopieloplastia, pieloplastia laparoscópica y pieloplastia robótica.

Clásicamente el tratamiento más extendido ha sido la pieloplastia a cielo abierto, hoy todavía es el "patrón oro" para esta patología ${ }^{2}$. En 1943 Davis describió por primera vez una ureterotomía ${ }^{5}$ y constituyó la base técnica de las modernas endopielotomías, que realizaron por primera vez en 1983 por Wickham y Kellet ${ }^{6}$. En el individuo adulto el tratamiento endourológico está aceptado universalmente, pues como refiere Motola et al es un procedimiento sencillo, seguro y eficaz; comparado con la cirugía abierta presenta: menor estancia hospitalaria, menor tiempo quirúrgico y menor convalecencia; si bien su tasa de éxito es algo inferior $88 \%$ vs $100 \%{ }^{7}$.

La causa más importante de hidronefrosis en la infancia es la EPU ${ }^{1,2}$, de ahí que se quiera aplicar la terapia endourológica a los enfermos de esta edad, intentando trasladar las ventajas de este abordaje del adulto al niño. El problema radica en que dada la heterogeneidad de este grupo, por edad y talla, es muy difícil hacer extensivo su uso de una forma generalizada. Así Rodrigues Netto et al. ${ }^{8}$ en un estudio comparativo entre pieloplastia vs endopielotomía anterógrada en adultos y en niños obtuvo que la pieloplastia en adultos aunque consigue mejores resultados que el procedimiento no invasivo (96 vs $82 \%$ ) presenta: mayor estancia hospitalaria, convalecencia y comorbilidad; pero en el grupo pediátrico la pieloplastia ofrece mejores resultados funcionales, como era de esperar, pero sorprendentemente además tiene una menor estancia hospitalaria y escasa comorbilidad por lo que concluye que es necesario un gran avance tecnológico en el instrumental, fabricándolo más pequeño y manejable, para que pueda ser competitivo.

Para valorar el tratamiento endourológico de la EPU en el niño es importante diferenciar: vía de acceso (anterógrada o retrógrada), edad del paciente y si es un tratamiento primario o secundario.

En primer lugar cuando valoramos los casos en los que el procedimiento endourológico es la primera opción terapéutica se constata la existencia de resultados muy heterogéneos. En nuestra serie existen dos casos primarios, uno resuelto vía anterógrada y otro vía retrógrada, ambos con buena evolución posterior. Emmanuel et al. compararon en una población de edad pediátrica diagnosticada de EPU y sin tratamiento previo, la pieloplastia abierta vs endopielotomía percutánea, resultando que el segundo grupo sólo mejoraba al primero en estancia hospitalaria ${ }^{2}$ (Tabla 2), y lo atribuye a que en este grupo los niños eran de mayor edad. Para Guy A. Bogaert con una población muy similar a la anterior comparó un grupo tratado mediante cirugía abierta y otro con endopielotomía, en este caso vía retrógrada, lográndose en el segundo grupo menor tiempo quirúrgico, coste, tiempo de hospitalización y analgesia que en el primero (Tabla 2). Para este autor la mayor limitación viene dada por la relación de tamaño entre el uréter y el instrumental, de hecho 4 niños requirieron una dilatación del meato ureteral, si bien para él es un procedimiento seguro ${ }^{9}$. Danuser $\mathrm{H}$. et al afirman que el tratamiento primario vía anterógrada de una 
Tabla 2

Resultados estudio de Emmanuel M. Schenkan et al. (filas 1y 2) y Guy A. Bogaert et al. (filas 3 y 4).

\begin{tabular}{lccccccc}
\hline & No & Edad & Éxito & Estancia & Tiempo quirúrgico & Coste & Analgesia \\
\hline Pieloplastia abierta & 20 & $1 \mathrm{~m}-25 \mathrm{a}$ & $93 \%$ & $3,3 \mathrm{~d}$ & $132 \mathrm{~min}$. & $5.931 \$$ & \\
Endopielotomía anterógrada & 8 & $2,5-19 \mathrm{a}$ & $88 \%$ & $2,5 \mathrm{~d}$ & $220 \mathrm{~min}$ & $8.474 \$$ & $11.560 \$$ \\
Pieloplastia abierta & 8 & $2 \mathrm{~m}-10 \mathrm{a}$ & & $2,1 \mathrm{~d}$ & $175 \mathrm{~min}$. & $\mathrm{mg}$ \\
Endopielotomía retrógrada & 8 & $4-15 \mathrm{a}$ & & $1 \mathrm{~d}$ & $119 \mathrm{~min}$ & $7.990 \$$ & $2.2 \mathrm{mg}$ \\
\hline
\end{tabular}

EPU, es efectivo $(88,7 \%)$ y no interfiere en una posible pieloplastia abierta posterior, por lo que recomienda hacer éste en primera instancia ${ }^{10}$. Por su parte Béla Tallai et al trataron 37 EPU en niños con edades comprendidas entre 4,5 y 17 años con un éxito del $81 \%$, y refiere que es un procedimiento con baja morbilidad y con un tiempo medio total de fluoroscopia de 210 segun$\operatorname{dos}^{11}$.

De un modo muy similar los resultados en los procedimientos secundarios son poco homogéneos, en nuestros 5 casos de EPU como tratamiento secundario, 2 han fracasado, si bien en el niño de 13 meses que presentó el episodio de pionefrosis (tratado con nefrostomía), creemos que la unidad renal se perdió realmente en este episodio y no por fracaso de la endopielotomía percutánea que se le practicó posteriormente. En la literatura está descrito en amplias series, con largo tiempo de evolución (seguimiento medio 88 meses), la existencia de buenos resultados (87\% éxito) para el procedimiento percutáneo en niños ya intervenidos previamente ${ }^{8}$, además muestran que el número de pieloplastias previas no influye en el resultado del procedimiento percutáneo posterior. Varios autores defienden el tratamiento endoscópico en EPU como tratamiento secundario tras una pieloplastia argumentando que al realizar ésta primero, en el momento de la pieloplastia se efectuará una transposición de los posibles vasos polares que estuviesen provocando esta patología, favoreciendo el resultado posterior de los procedimientos que emplean la vía endoluminal como vía de abordaje ${ }^{5}$. Además dado que el $33-60 \%$ de pieloplastias que se reintervienen con cirugía a cielo abierto, debido a la dificultad técnica que presenta, acaban en nefrectomía ${ }^{8,12,13}$ se defiende el tratamiento endourológi$\mathrm{co}^{14}$ como segunda opción terapéutica.
John S. Lam et al. en 93 niños en edad pediátrica, con un seguimiento medio de 32 meses comparó el acceso anterógrado y el retrógrado, obteniéndose un mayor éxito con la vía anterógrada ( $81 \%$ vs $75 \%$ ); en tiempo quirúrgico, estancia hospitalaria y dolor; es de tener en cuenta el hecho de que con el acceso retrógrado consiguió mejores resultados funcionales ${ }^{15}$.

El acceso percutáneo suele emplearse más en caso de que la población sea de corta edad por un problema de tamaño del instrumental. En la Universidad de Washington utilizaron este acceso en 17 niños, y compararon los resultados en función de si pertenecían a tratamiento primario o secundario. Lo más llamativo del mismo es la diferencia de éxito entre ambos grupos: $62 \%$ el primario y $100 \%$ el secundario (siendo la media de edad de 12 vs 4 años), si bien consiguió menor tiempo quirúrgico (102 vs 171 minutos) y estancia (3 vs 6 días) al acceder retrógradamente ${ }^{6}$. Hay publicaciones que presentan mucho peores tasas de éxito $67 \% \%^{5}$, pero atribuyen estos resultados a que en otros estudios aportan el estado actual del paciente por encuesta telefónica y alegan que ellos en sus resultados obtienen hasta un 30\% de fracasos terapéuticos en enfermos absolutamente asintomáticos (en una encuesta telefónica no serían detectados). Además el tiempo de seguimiento en la mayoría de los estudios es inferior a doce meses y ellos tienen un largo seguimiento que varía de 16-138m (con una media 55 meses) y descubren el $33 \%$ de sus fracasos pasado el primer año, incluso están descritos pasados 36 meses $^{12}$.

Generalizando, la mayoría de los autores están de acuerdo en que hay muchos factores que se relacionan con la obtención de buenos resultados en los procedimientos endoscópicos: grado de dilatación, tamaño pelvis redundante, 
grosor cortical renal, función renal previa, estenosis de largo trayecto y vasos polares ${ }^{8,16}$. La experiencia del cirujano, la buena selección del enfermo y la disponibilidad de material adecuado son la clave para lograr el éxito en esta nueva empresa.

\section{CONCLUSIÓN}

El tratamiento endourológico para la EPU en la edad pediátrica es factible. Dada la heterogeneidad de resultados publicados en la literatura parece claro que la experiencia del cirujano, la disponibilidad de material adecuado y la correcta selección del enfermo son las claves para lograr un tratamiento exitoso. La elección de la vía de abordaje y si el procedimiento debe ser primario o secundario debe definirse con el desarrollo de más estudios y más amplios. La investigación y el desarrollo de nuevo material endourológico serán los que finalmente universalicen la realización de estas técnicas como "técnicas de rutina" en todos los centros.

\section{REFERENCIAS}

1. Gomez Fraile A, Aransay Brantot A, Delgado Muñoz D, López Vázquez F, Serrano Hernandez C, Manrique Legaz A Is it necessary to use an isotopic diuretic renogram in grade II pyelocaliceal dilatations diagnosed with ultrasonography?. Actas Urol Esp. 2001;25(1):60-63.

2. Schenkman EM, Tarry WF. Comparison of percutaneous endopyelotomy with open pyeloplasty for pediatric ureteropelvic junction obstruction. J Urol. 1998;159(3):1013-1015.

3. O’Reilly PH. Diuresis renography, recent advances and recommended protocols. Br J Urol. 1992;69(2):113-120.

4. Ransley PG, Dhillon HK, Gordon I, Duffy PG, Dillon MJ, Barrat TM. The postnatal management of hydronefrosis diagnosed by prenatal ultrasound. J Urol. 1990;144(2 Pt 2):584-587; disscusion 593-594.

5. Tawfiek ER, Liu JB, Bagley DH. Ureteroscopic treatment of ureteropelvic junction obstruction. J Urol. 1998;160(5): 1643-1646.
6. Fingenshau RS, Clayman RV, Colberg JW, Coplen DE, Soble JJ, Manley CB. Pediatric endopyelotomy: the Washington University experience. J Urol. 1996;156(6): 2025-2030.

7. Desai MM, Desai MR, Gill IS. Endopyeloplasty versus laparoscopic pyeloplasty for primary ureteropelvic junction obstruction. Urology. 2004;64(1):16-21; disscusion 21.

8. Rodrigues Netto N, Ikari O, Esteves SC, D’Ancona CA. Antegrade endopyelotomy for pelvi-ureteric junction obstruction in children. Br J Urol. 1996;78(4):607-612.

9. Bogaert GA, Kogan BA, Mevorach RA, Stoller ML. Efficacy of retrograde endopyelotomy in children. J. Urol., 1996;156(2 Pt 2):734-737.

10. Knudsen BE, Cook AJ, Watterson JD, Beiko DT, Nott L, Razvi $\mathrm{H}$, et al. Percutaneous antegrade endopyelotomy: long term results from one institution. Urology. 2004; 63(2):230-234.

11. Tallai B, Salah MA, Flasko T, Toth C, Varga A. Endopyeltomy in childhood: our experience with 37 patients. J Endourol. 2004;18(10):952-958.

12. Lam JS, Cooper KL, Greene TD, Gupta M. Impact of hydronephrosis and renal function on treatment outcome: antegrade versus retrograde endopyelotomy. Urology. 2003; 61(6):1107-1111; discussion 1111-1112.

13. Albani JM, Yost AJ, Streem SB. Ureteropelvic junction obstruction: determining durability of endourological intervention. J Urol. 2004;171(2 Pt 1):579-82.

14. Jabbour ME, Goldfischer ER, Klima JW, Stravodimos KG, Smith AD. Endopyelotomy after failed pyeloplasty: the long-term results. J Urol. 1998; 160 (3 Pt 1):690-692; discussion 692-693.

15. Danuser H, Ackerman DK, Bohlen D, Studer UE. Endopyelotomy for primary ureteropelvic junction obstruction risk factors determine the success rate. J Urol. 1998; 159(1):56-61.

16. Figenshau RS, Clayman RV. Endourologic options for management of ureteropelvic junction obstruction in the pediatric patient. Urol Clin North Am. 1998;25(2):199-209.

Correspondencia autor: Dr. J. Romero Otero

Servicio de Urología. Hospital Universitario 12 de Octubre. Avda. de Córdoba, s/n. 28041 Madrid. Tel.: 913908121 E-mail autor: jromerootero@hotmail.com

Información artículo: Original - Urología pediátrica

Trabajo recibido: octubre 2006

Trabajo aceptado: noviembre 2006 